\title{
LncRNA THRIL is involved in the proliferation, migration, and invasion of rheumatoid fibroblast-like synoviocytes
}

\author{
Yaoyao Zou ${ }^{1 \#}$, Chuyu Shen ${ }^{2 \#}$, Ting Shen ${ }^{3}$, Jingnan Wang ${ }^{2}$, Xuepei Zhang ${ }^{1}$, Qian Zhang ${ }^{1}$, Runlu Sun ${ }^{4}$, \\ Lie Dai ${ }^{1}$, Hanshi Xu ${ }^{2}$ \\ ${ }^{1}$ Department of Rheumatology, Sun Yat-sen Memorial Hospital, Sun Yat-sen University, Guangzhou, China; ${ }^{2}$ Department of Rheumatology and \\ Immunology, The First Affiliated Hospital, Sun Yat-sen University, Guangzhou, China; ${ }^{3}$ Department of Radiation Oncology, Sun Yat-sen Memorial \\ Hospital, Sun Yat-sen University, Guangzhou, China; ${ }^{4}$ Department of Cardiology, Sun Yat-sen Memorial Hospital, Sun Yat-sen University, \\ Guangzhou, China \\ Contributions: (I) Conception and design: Y Zou, H Xu, L Dai; (II) Administrative support: H Xu; (III) Provision of study materials or patients: H Xu, \\ C Shen; (IV) Collection and assembly of data: Y Zou, X Zhang, Q Zhang, T Shen; (V) Data analysis and interpretation: Y Zou, C Shen, J Wang, R \\ Sun; (VI) Manuscript writing: All authors; (VII) Final approval of manuscript: All authors. \\ \#These authors contributed equally to this work. \\ Correspondence to: Hanshi Xu. Department of Rheumatology and Immunology, The First Affiliated Hospital, Sun Yat-sen University, 58 Zhongshan \\ Road 2, Guangzhou 510080, China. Email: xuhanshi@mail.sysu.edu.cn; Lie Dai. Department of Rheumatology, Sun Yat-sen Memorial Hospital, Sun \\ Yat-sen University, 107 Yan Jiang West Road, Guangzhou 510120, China. Email: dailie@mail.sysu.edu.cn.
}

Background: Fibroblast-like synoviocytes (FLSs), which can migrate and directly invade the cartilage and the
bone, are crucial players in joint damage in rheumatoid arthritis (RA). Nevertheless, the detailed mechanisms
underlying the aberrant activation of RA FLSs remain unclear. Several studies have attempted to explore the
relationship between long non-coding RNAs (lncRNAs) and RA pathology; however, the role of lncRNAs in RA
is unknown. The present study aimed to determine the functions of tumor necrosis factor- $\alpha$ and heterogeneous
nuclear ribonucleoprotein L-related immunoregulatory lincRNA (THRIL) in RA FLSs migration and invasion.
Methods: Small interfering RNA targeting THRIL or lentivirus overexpressing THRIL was used to knockdown
or overexpress THRIL. Quantitative reverse transcription polymerase chain reaction (PCR) was employed for the
detection of RNA expression. The proliferation rate of RA FLSs was measured using a 5-ethynyl-2'-deoxyuridine
(EdU) incorporation assay. Migration and invasion were detected using a transwell chamber. Downstream targets
were identified using a human cell cycle real-time PCR array and a human cell motility real-time PCR array.
Results: A significant decrease in THRIL expression was found in RA FLSs compared with cells from
healthy control (HC)patients. THRIL is mainly localized in the nucleus. Knockdown of THRIL increased
the proliferation, migration, and invasion of RA FLSs. In contrast, THRIL overexpression had the opposite
effect. THRIL knockdown increased interleukin-1 $\beta$ (IL-1 3 )-triggered expression of matrix metalloproteinase
(MMP)-1, MMP-3, and $M M P-13$. THRIL overexpression led to a significant decrease in $M M P-13$ expression
in response to stimulation with IL-1 3 . Furthermore, we observed that the expression levels of cyclin-
dependent kinase 1 (CDK1) and G2 and S phase-expressed-1 (GTSE1), both of which are associated with
cellular mobility and proliferation, were downregulated with THRIL overexpression.

Conclusions: Reduced expression of lncRNA THRIL represses the proliferation, migration, and invasion of RA FLSs, suggesting that lncRNA THRIL might be a potential target for RA therapy.

Keywords: Rheumatoid arthritis (RA); long non-coding RNA (lncRNA); migration; invasion

Submitted Mar 26, 2021. Accepted for publication Jun 20, 2021.

doi: $10.21037 / \mathrm{atm}-21-1362$

View this article at: https://dx.doi.org/10.21037/atm-21-1362

^ ORCID: 0000-0001-9493-9032. 


\section{Introduction}

As one of the most prevalent systemic rheumatoid diseases, rheumatoid arthritis (RA), which is characterized by synovitis and progressive joint damage, has been perceived as a major cause of extremity disability $(1,2)$. To date, however, the etiology of RA has not yet been fully clarified. It has been well established that fibroblast-like synoviocytes (FLSs), located in the highly inflamed and invasive RA pannus, play vital roles in RA pathology (3-5). Previous studies have demonstrated that RA FLSs exhibit enhanced secretion of various proinflammatory cytokines, chemokines, and matrix metalloproteinases (MMPs) that participate in inflammatory infiltration and joint destruction. Interestingly, RA FLSs display an aggressive phenotype that surprisingly resembles that of tumor cells, including uncontrolled proliferation, resistance to apoptosis, invasiveness to cartilage, and migration to unaffected joints $(3,5)$. Considerable research has been devoted to exploring the mechanisms underlying the "tumor-like transformation" of RA FLSs, but these mechanisms are still largely unknown. Therefore, identifying key regulators that control the pathogenetic behaviors of RA FLSs could shed light on novel FLS-targeted therapy for RA.

The past 2 decades have seen dramatic development in non-coding RNA research, especially in long non-coding RNAs (lncRNAs). Increasing evidence indicates that lncRNAs participate in regulating various cellular biological processes (6-8) and initiating and developing various diseases, including RA (9-11). Attempts have been made to investigate the function of lncRNAs in regulating the aberrant behaviors displayed by RA FLSs. Our previously published work identified a novel downregulated lncRNA, lowly expressed in rheumatoid fibroblast-like synoviocytes (LERFS), which suppresses the proliferation, migration, and invasion of RA FLSs by abrogating the expression of small GTPase proteins through interaction with hnRNP Q (12). In addition, lncRNA Fer-1-like protein 4 (FER1L4) has been found to modulate synovial inflammation by targeting nucleotide oligomerization domain-like receptors 5 (NLRC5) (13). However, several lncRNAs, including ZNFX1 antisense RNA 1 (ZFAS1) (14), gastric adenocarcinoma associated, positive CD44 regulator, long intergenic non-coding RNA (GAPLINC) (15), and P38 inhibited cutaneous squamous cell carcinoma associated lincRNA (PICSAR) (16), are involved in modulating the activated phenotype of RA by working as competing endogenous RNAs through sponging microRNAs. To date, accumulating evidence indicates that lncRNAs could be pivotal regulators and promising therapeutic targets in RA; however, little is currently known about the function of lncRNAs on synovial inflammation and articular damage.

Recently, lncRNA tumor necrosis factor- $\alpha$ (TNF- $\alpha$ ) and heterogeneous nuclear ribonucleoprotein L (hnRNPL)related immunoregulatory lincRNA (THRIL) has shown crucial regulatory effects on inflammation and immune response. For instance, THRIL was found to be required for the expression of a wide variety of cytokines, especially TNF- $\alpha$, by interacting with hnRNPL to form a functional complex that binds to the promoter of target genes in human macrophages. In addition, the expression level of THRIL is associated with the severity of Kawasaki disease, an autoimmune-related vasculitis of children (17). T cells isolated from RA patients display increased expression of THRIL compared with T cells from healthy individuals (18).

Interestingly, a recent study reported the possible involvement of THRIL in regulating RA FLS growth and inflammatory response (19). However, it is still unclear whether THRIL is involved in modulating the transformed and invasive phenotype of RA FLSs. Therefore, in the present study, we primarily focused on the involvement of THRIL in the migration and invasion behaviors displayed by RA FLSs. We present the following article in accordance with the MDAR reporting checklist (available at https:// dx.doi.org/10.21037/atm-21-1362).

\section{Methods}

\section{Preparation of human specimens}

We used synovial tissue (ST) specimens from RA patients with active disease $(n=10,7$ females and 3 males; age range: 46-61 years) and osteoarthritis patients $(n=5,3$ females and 2 males; age range: 54-66 years) who underwent synovectomy, joint replacement of the knee joint, or Parker-Pearson needle synovial biopsy at the First Affiliated Hospital, Sun Yat-sen University, China. All RA patients were diagnosed based on the 2010 ACR/EULAR criteria for RA classification (20). STs of healthy controls (HCs) $(n=9,6$ females and 3 males; age range: 32-66 years) were obtained from individuals who underwent orthopedic surgery because of trauma, meniscus injury, or cruciate ligament injury. Individuals with manifestations of acute or chronic arthritis were not included. The study was performed in compliance with the Declaration of Helsinki (as revised in 2013). The Medical Ethics Committee of the 
Table 1 Primers used in this study for the detection of the indicated RNAs

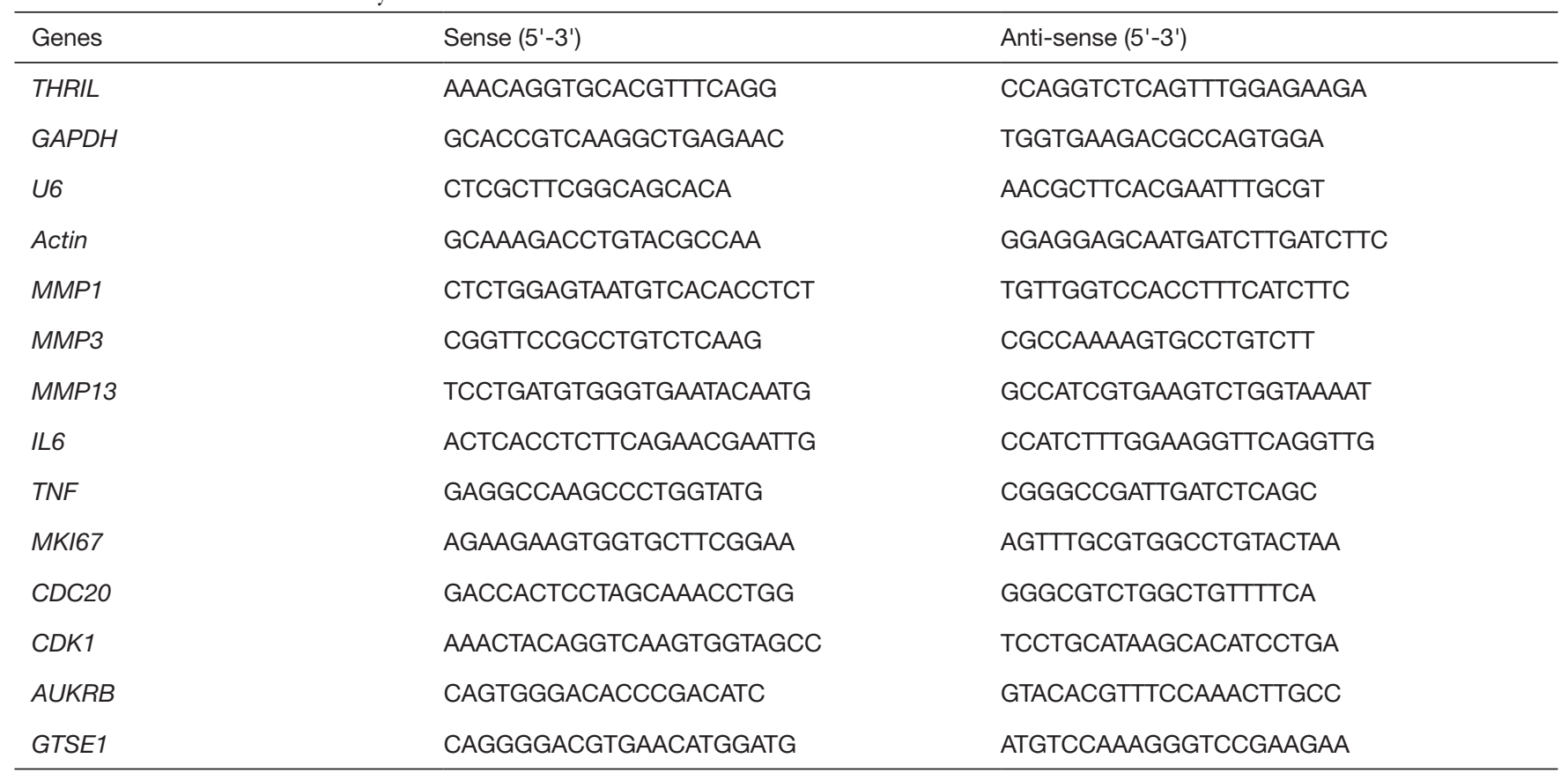

AUKRB, aurora kinase B; CDC20, cell division cycle 20; CDK1, cyclin-dependent kinase 1; GAPDH, glyceraldehyde-3-phosphate dehydrogenase; GTSE1, G2 and S phase-expressed-1; IL-6, interleukin 6; MKI67, markers of proliferation Ki-67; MMP, matrix metalloproteinase; THRIL, tumor necrosis factor- $\alpha$ and heterogeneous nuclear ribonucleoprotein L-related immunoregulatory lincRNA; $T N F$, tumor necrosis factor.

First Affiliated Hospital of Sun Yat-sen University approved all research protocols involved in this research \{No. [2017]049\}. All participants provided written informed consent.

\section{Cell isolation and culture}

ST specimens were cut into 1-mm-diameter pieces and washed with phosphate-buffered saline (PBS). After digestion for $2 \mathrm{~h}$ at $37^{\circ} \mathrm{C}$ with $1 \mathrm{mg} / \mathrm{mL}$ type I collagenase (Sigma-Aldrich, St. Louis, MO, USA), isolated RA FLSs were washed with PBS and then resuspended and cultured adherently in Dulbecco's modified Eagle's medium with $10 \%$ fetal bovine serum (FBS) under $5 \% \mathrm{CO}_{2}$ at $37{ }^{\circ} \mathrm{C}$. RA FLSs from passages 4-6 were used in subsequent experiments.

\section{Separation of nuclear and cytoplasm fractions}

The nuclear and cytoplasmic fractions of FLSs were separated using a PARIS kit (Thermo Fisher Scientific, Waltham, MA, USA). In brief, RA FLSs at $90 \%$ confluence were trypsinized and lysed using cell fractionation buffer. After collecting the supernatant, the remaining nuclear pellet was washed and further lysed, and the nuclear fraction was collected.

\section{Extraction of RNA and quantitative reverse transcription polymerase chain reaction ( $q R T-P C R$ )}

Total RNA was extracted with TRIzol reagent (Thermo Fisher Scientific, Waltham, MA, USA), and the isolated RNA was quantified with a NanoDrop 2000 (Thermo Fisher Scientific, Waltham, MA, USA). Reverse transcription of RNA was carried out with a PrimeScript RT kit (Takara, Tokyo, Japan). A SYBR Premix Ex Taq kit (Takara, Tokyo, Japan) was used for qRT-PCR, and the Bio-Rad CFX96 system (Bio-Rad, Hercules, CA, USA) was employed. Details of the primers used in amplification are provided in Table 1. The PCR program was set as follows: $95{ }^{\circ} \mathrm{C}$ for $30 \mathrm{~s}$, followed by 40 cycles of $95^{\circ} \mathrm{C}$ for $5 \mathrm{~s}$ and then $60^{\circ} \mathrm{C}$ for $30 \mathrm{~s}$. GAPDH was used as the internal reference gene, and the $2^{-\Delta \Delta \mathrm{Ct}}$ method was employed to 
Table 2 siRNA sequence targeting THRIL

\begin{tabular}{ll}
\hline Sequences & Target sequence for THRIL siRNAs \\
\hline siTHRIL-001 & CCACCAATCCCTAAGCTGT \\
siTHRIL-002 & GTGTGTAGTTCCACGTCAA \\
siTHRIL-003 & GCCACTTCTTGCTCAGTC \\
Negative control siRNA & $\begin{array}{l}\text { Purchased from RiboBio } \\
\text { (siN0000001-1-5) }\end{array}$ \\
\hline
\end{tabular}

siRNA, small interfering RNA; THRIL, tumor necrosis factor- $\alpha$ and heterogeneous nuclear ribonucleoprotein L-related immunoregulatory lincRNA.

calculate gene expression.

\section{Transfection of small interfering RNA (siRNA)}

siRNAs targeting THRIL, scramble control siRNA, and CY3-labeled siRNA were synthesized at RiboBio (Guangzhou, China). RA FLSs at 70\% confluence were transfected with the indicated siRNA with Lipofectamine RNAiMAX (Thermo Fisher Scientific, Waltham, MA, USA). The target sequences of the indicated genes are shown in Table 2.

\section{Infection of overexpression lentivirus}

Lentiviruses overexpressing THRIL and vector control lentiviruses were constructed at GeneChem (Shanghai, China). Cells at $60 \%$ confluence were infected with lentivirus particles $(\mathrm{MOI}=30)$ in the presence of $10 \mu \mathrm{g} / \mathrm{mL}$ polybrene.

\section{EdU incorporation assay}

The Cell-Light EdU Apollo567 In Vitro Kit (RiboBio, Guangzhou, China) was utilized to evaluate the proliferation rate. FLSs at a confluence of $80 \%$ were incubated with EdU solution $(1: 1,000)$ for $8 \mathrm{~h}$ and then subjected to EdU and Hoechst staining. EdU-positive cells were regarded as proliferating cells and counted under a fluorescence microscope.

\section{Apoptosis assay}

The cell apoptosis rate of FLSs was measured by annexin $\mathrm{V}$ and 7-AAD staining. Briefly, FLSs were washed with PBS and then suspended in $200 \mu \mathrm{L} 1 \times$ binding buffer;
$5 \mu \mathrm{L}$ annexin V-APC (BD Bioscience, San Jose, CA, USA) was added to the cell suspension and incubated for $15 \mathrm{~min}$ at room temperature away from light. Then $5 \mu \mathrm{L}$ 7-AAD (BD Biosciences, San Jose, CA, USA) was added, and the samples were analyzed by flow cytometry.

\section{Migration and invasion assay}

Transwell inserts (Corning, NY, USA) with $8 \mu \mathrm{m}$ pores were used to evaluate migration. Briefly, after counting and resuspending in serum-free medium, RA FLSs $\left(2.5 \times 10^{4} /\right.$ well) were seeded into transwell inserts, and cell culture medium supplemented with $10 \%$ FBS was added to the wells below the chambers for directed chemotaxis. Six hours later, after removing the cells remaining above the transwell membrane using cotton swabs, RA FLSs that migrated across the transwell membrane were visualized by crystal violet staining and counted manually under an optical microscope (magnification 100x). To detect the invasion rate, transwell chambers precoated with Matrigel (BD Biosciences, San Jose, CA, USA) were utilized, $5 \times 10^{4}$ cells were seeded, and a medium with $15 \%$ FBS was used as a chemoattractant. After $30 \mathrm{~h}$, the number of invading cells was counted as described for the migration assay.

\section{qRT-PCR array}

To identify target genes regulated by THRIL, RT2 Profiler PCR Array Human Cell Cycle and RT ${ }^{2}$ Profiler PCR Array Human Cell Motility (Kang Chen Biotech, Shanghai, China) were used. In brief, RNA of RA FLSs was extracted with TRIzol and cleaned using the RNeasy MinElute Cleanup Kit (Qiagen, Duesseldorf, Germany) to remove contaminating DNA. RNA was then reverse transcribed to produce cDNA using the RT2 First Strand Kit (Invitrogen, Carlsbad, CA, USA) and amplified by PCR using RT2 SYBR Green qPCR Master Mix (Invitrogen, Carlsbad, CA, USA). Five housekeeping genes, including glyceraldehyde3-phosphate dehydrogenase $(G A P D H)$, actin beta $(A C T B)$, ribosomal protein lateral stalk subunit P0 (RPLPO), hypoxanthine phosphoribosyltransferase 1 (HPRT1), and beta-2-microglobulin (B2M), were incorporated in the assay, and the average $\mathrm{Ct}$ values were calculated.

\section{Statistical analysis}

GraphPad Prism 8 for Windows (GraphPad, La Jolla, CA, USA) was used to carry out statistical analyses and draw 


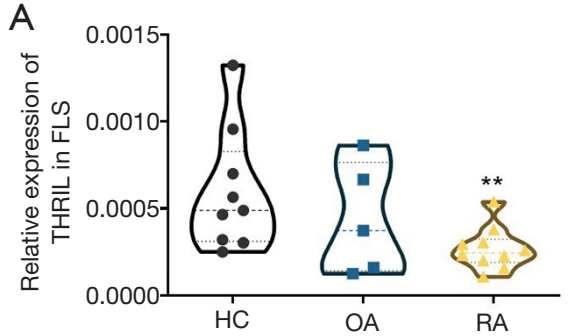

C

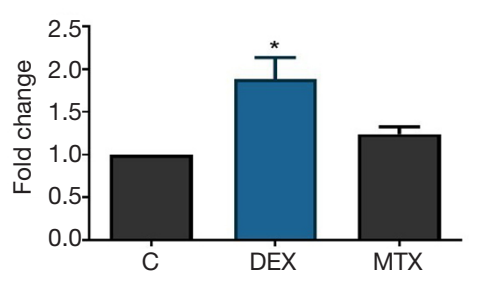

B
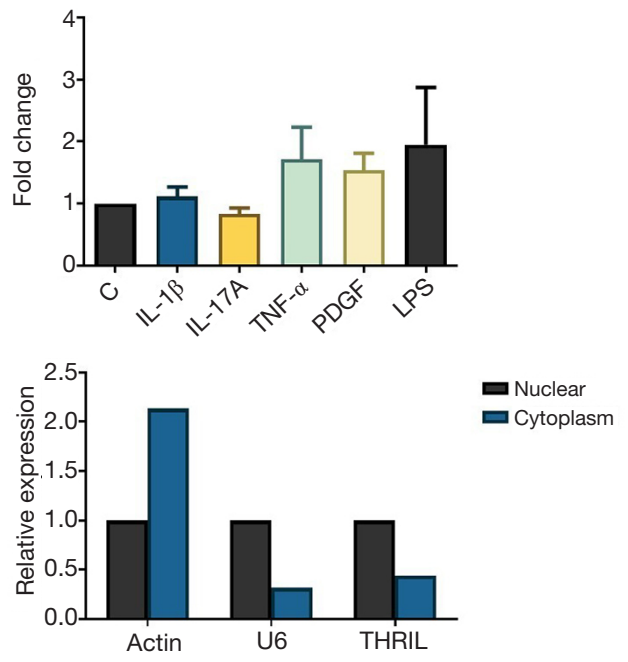

Figure 1 Expression of tumor necrosis factor- $\alpha$ and heterogeneous nuclear ribonucleoprotein L-related immunoregulatory lincRNA (THRIL) in rheumatoid arthritis (RA) fibroblast-like synoviocytes (FLSs) and healthy control (HC) FLSs. (A) Differential expression of THRIL in FLSs from HCs ( $n=9)$, osteoarthritis (OA) patients $(\mathrm{n}=5)$ and RA patients $(\mathrm{n}=10)$. $\bullet$ HC individuals, $\bullet \mathrm{OA}$ patients, $\boldsymbol{\Delta} \mathrm{RA}$ patients. ${ }^{* *} \mathrm{P}<0.01$ vs. HC. (B) Expression of THRIL under treatment with various cytokines. RA FLSs were stimulated with interleukin (IL)-1 $\beta$ (10 ng/mL), IL-17A (10 ng/mL), TNF- $\alpha(10 \mathrm{ng} / \mathrm{mL})$, platelet derived growth factor(PDGF) $(10 \mathrm{ng} / \mathrm{mL})$, or Lipopolysaccharide (LPS) (100 ng/mL) for 24 h. (C) Effect of dexamethasone (DEX) or methotrexate (MTX) on THRIL expression in RA FLSs. RA FLSs were treated with DEX $(1 \mu \mathrm{g} / \mathrm{mL})$ or MTX $(10 \mu \mathrm{g} / \mathrm{mL})$ for $24 \mathrm{~h}$. Ct values were normalized to glyceraldehyde-3-phosphate dehydrogenase $(G A P D H)$. Data shown are the mean \pm standard error of mean from at least 3 independent experiments. ${ }^{*} \mathrm{P}<0.05$ vs. control (C). (D) THRIL expression in cytoplasmic and nuclear fraction of FLSs. Actin served as a cytoplasmic marker and U6 served as a nuclear marker.

statistical charts. The values shown were pooled from at least 3 independent experiments and presented as the mean value \pm standard error of the mean. To minimize variability between independent experiments using FLSs from different RA patients, some data were normalized and presented as the fold change over the indicated control group. A 2-tailed Student's $t$-test was employed for comparisons between 2 groups. For comparisons between 3 or more different groups, 1-way analysis of variance with Bonferroni's post-hoc test was employed. $\mathrm{P}<0.05$ was considered to be statistically significant.

\section{Results}

\section{Decreased expression of IncRNA THRIL in FLSs from RA patients}

First, qRT-PCR was employed for the quantification of THRIL expression in FLSs. As shown in Figure 1A, RA FLSs displayed significantly decreased expression of THRIL compared with HC FLSs. It is now well established that pro-inflammatory cytokines are key components that form the inflammatory microenvironment in the rheumatoid synovium. We evaluated the influences of cytokine stimulation on the expression of THRIL in RA FLSs. However, unexpectedly, THRIL expression in RA FLSs was not influenced by stimulation with interleukin (IL)-1 $\beta$, IL-17A, TNF- $\alpha$, PDGF, or LPS for $24 \mathrm{~h}$ (Figure 1B). In addition, treatment with dexamethasone, but not methotrexate, increased THRIL expression in RA FLSs (Figure 1C).

Next, to explore the expression pattern and intracellular localization of THRIL, we separated the nuclear and cytoplasmic fractions of RA FLSs. We found that THRIL was primarily expressed in the nucleus (Figure 1D), suggesting that THRIL probably exerts its regulatory function at the transcription level in RA FLSs, as previously reported for macrophages (17).

\section{THRIL is a negative regulator of RA FLS proliferation}

siRNAs targeting THRIL were transfected into RA FLSs, and the silencing efficiency was confirmed (Figure 2A,2B). 
A BF

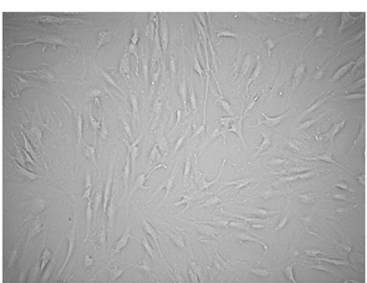

C

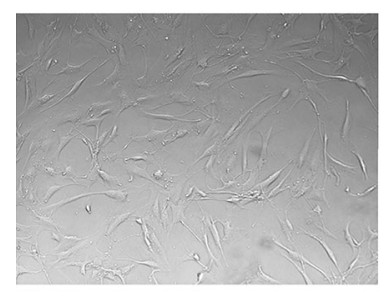

CY3

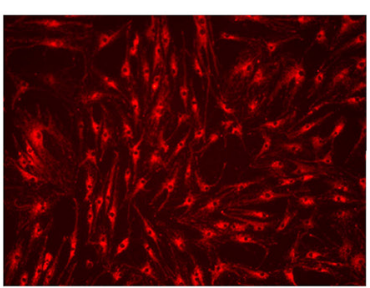

EGFP

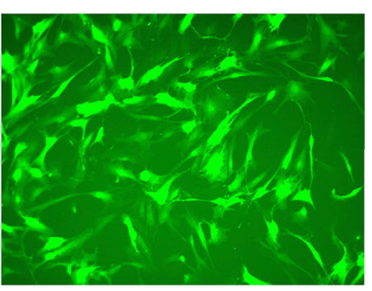

MERGE

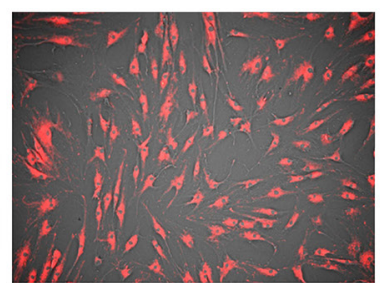

MERGE

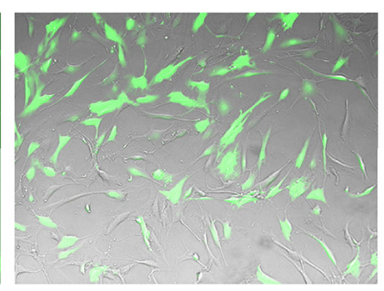

B

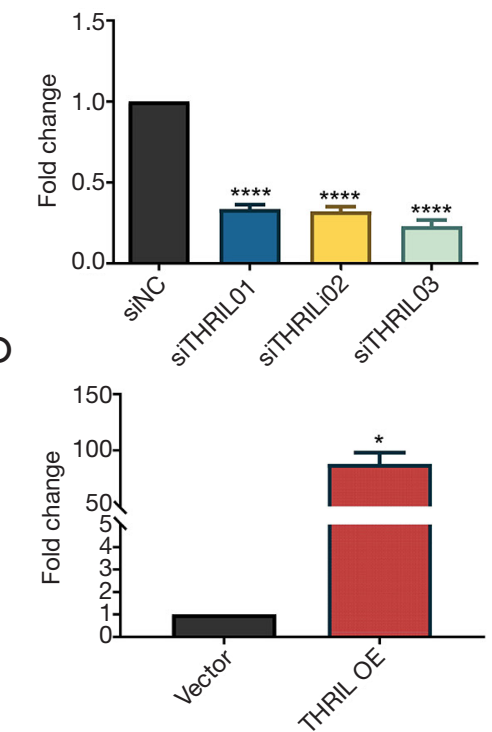

Figure 2 Efficiency of tumor necrosis factor- $\alpha$ and heterogeneous nuclear ribonucleoprotein L-related immunoregulatory lincRNA (THRIL) knockdown or overexpression in rheumatoid arthritis (RA) fibroblast-like synoviocytes (FLSs). (A) CY3-labeled small interfering RNA (siRNA) was transfected to RA FLSs at a final concentration of $50 \mathrm{nM}$ and visualized under a fluorescence microscope after 48 . Red fluorescence represents CY3-labeled siRNA. Original magnification 100×. (B) RA FLSs were transfected with scramble siRNA (siNC) or THRIL siRNA (siTHRIL), and efficiency of THRIL silencing was determined by quantitative reverse transcription polymerase chain reaction (qRT-PCR). (C) RA FLSs were infected with Green Fluorescent Portein (GFP)-carrying lentivirus at a Multiplicity of Infection (MOI) of 30 and observed after $72 \mathrm{~h}$. Green fluorescence represents GFP-positive cells. Original magnification 100×. (D) Expression level of THRIL in RA FLSs after infection with empty vector lentivirus (Vector) or THRIL overexpression lentivirus (THRIL OE) was measured by qRTPCR. Data shown are the mean \pm standard error of mean from at least 3 independent experiments. ${ }^{*} \mathrm{P}<0.05,{ }^{* * * *} \mathrm{P}<0.0001$ vs. control small interfering RNA (siNC) or Vector.

Transfection of all 3 siRNAs successfully decreased the expression level of THRIL, and siTHRIL03, which showed the greatest silencing efficiency, was chosen for the subsequent experiments. A lentivirus-based overexpression strategy was employed to increase the expression of THRIL (Figure 2C,2D).

Because the significant proliferation and reduced apoptosis of RA FLSs contribute to synovial hypertrophy in the articular cavity, we investigated the role of THRIL in controlling the proliferation rate of RA FLSs. We found that silencing THRIL with siRNA significantly increased the percentage of proliferating cells, as measured by EdU staining (Figure 3A). In contrast, as shown in Figure $3 B$, THRIL overexpression by lentivirus markedly decreased the proliferation rate compared with that of the empty vector control in RA FLSs. However, we observed that the proliferation of HC FLSs with THRIL knockdown or overexpression was not different from that of the control
siRNA or empty vector control group (Figure $3 C, D$ ). We further demonstrated that THRIL knockdown or overexpression did not influence the apoptosis of RA FLSs (Figure $3 E, 3 F$ ) and HC FLSs (Figure 3G,3H).

\section{THRIL represses the migration and invasion of $R A$ FLSs}

Another key pathogenic feature of RA FLSs is tumor-like aggressive behavior characterized by enhanced migration and invasion ability, which is the main cause of bone and cartilage destruction in joints. To establish whether the aberrant expression of THRIL modulates the migration of RA FLSs, we performed a transwell assay to measure chemotaxis migration. We found that siRNA-mediated knockdown of THRIL promoted the migration of RA FLSs (Figure $4 A$ ). We then evaluated the influence of THRIL on RA FLSs invasion through a transwell chamber precoated with Matrigel. The findings indicated that knockdown of 


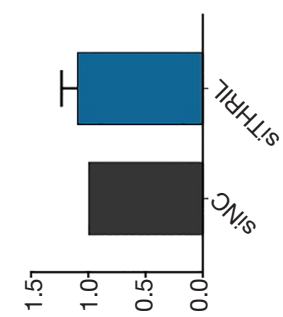

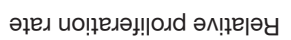
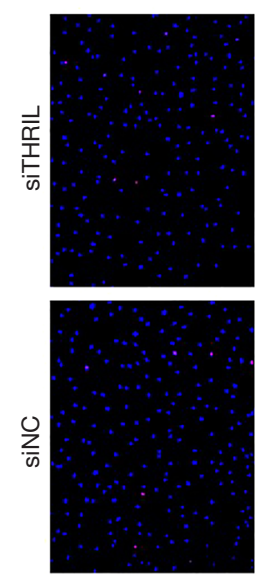

‡sчәәон/กดヨ

U

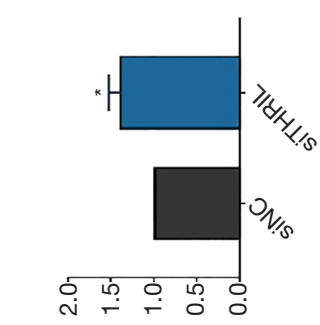

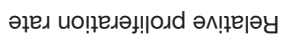
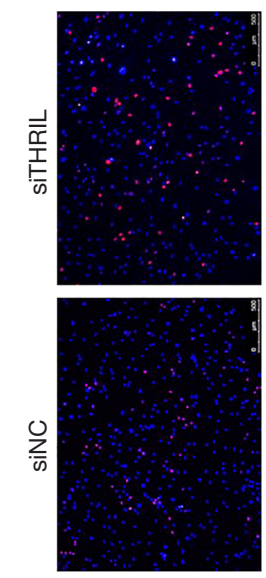

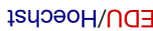

$\varangle$

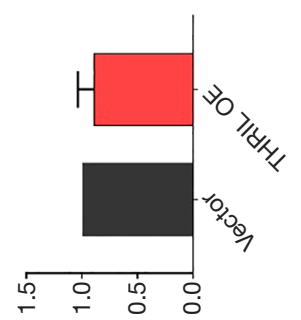

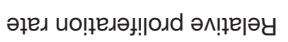

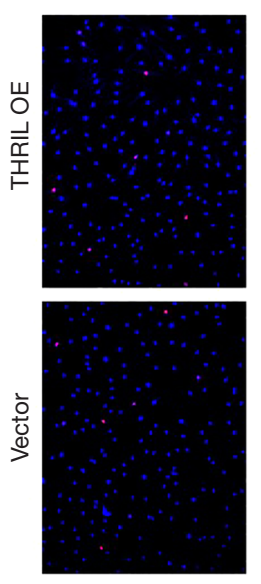

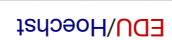

口

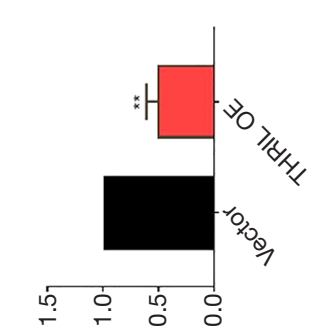

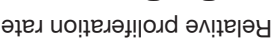
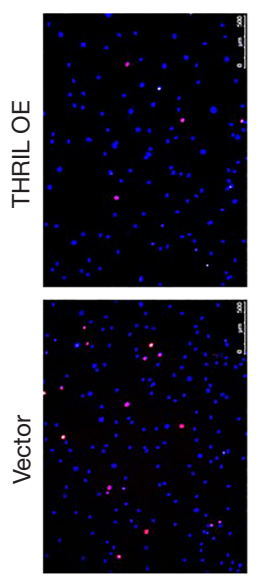

เงчวәон/กด

$\infty$

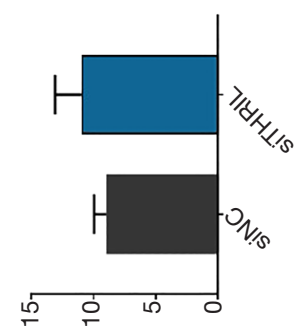

라소 s!soldode 타이
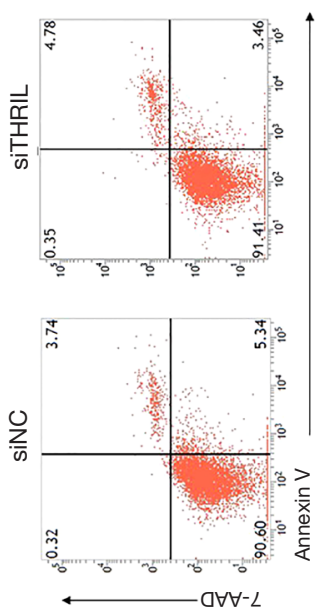

৩

I
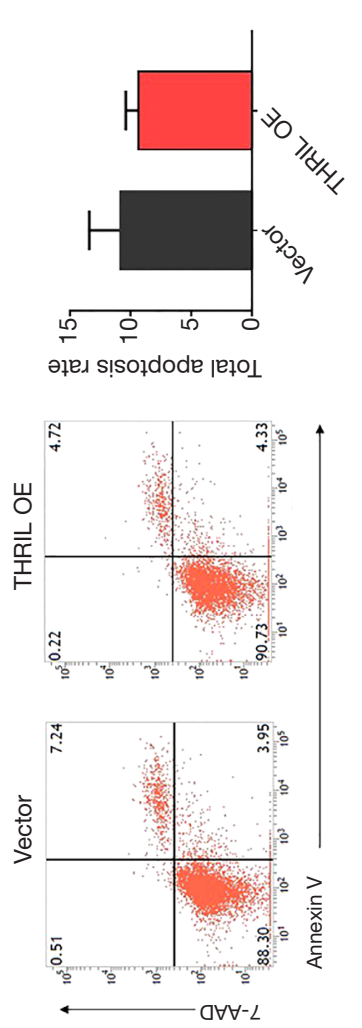

캇 s!soldode ן리니

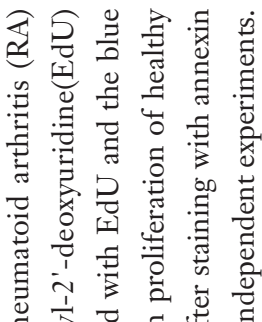

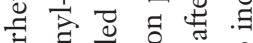

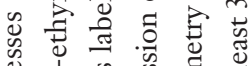

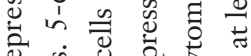

एँ

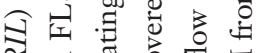

$\leqslant \varangle$ एव

舟语

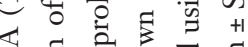

茓.

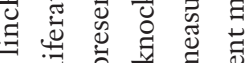

过可

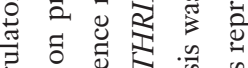

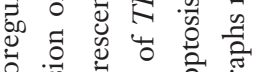

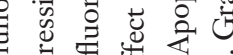

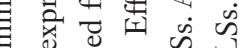

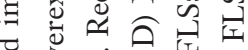

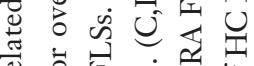

过故出

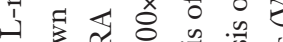

更

एँ

के

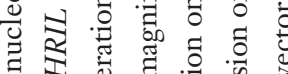

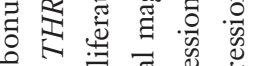

士

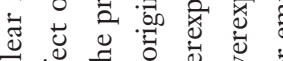

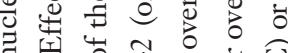

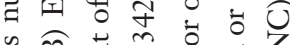

党
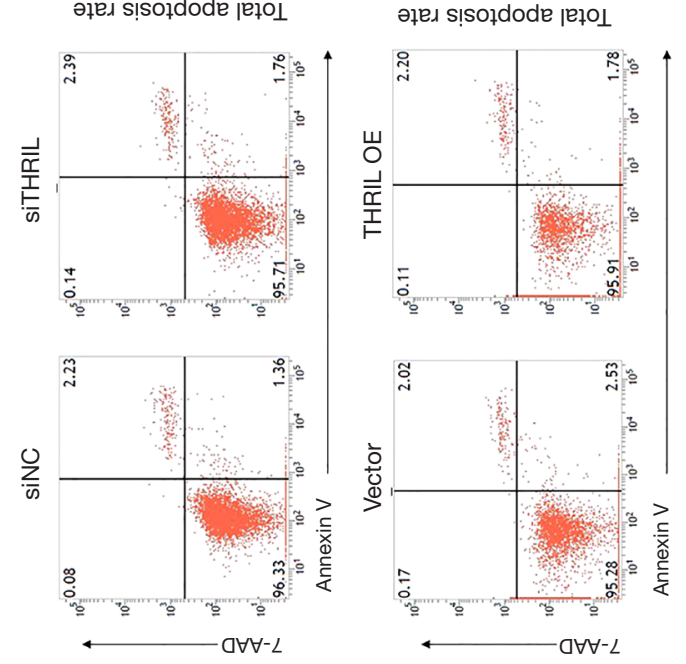

Ш

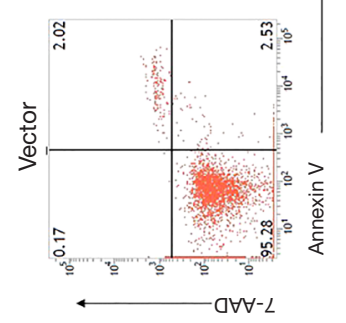

ㄴ.

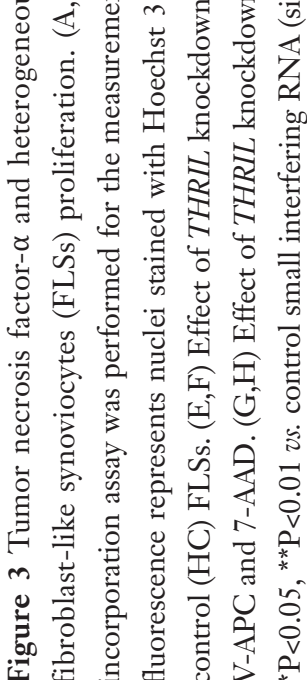


THRIL significantly increased the invasion of RA FLSs (Figure 4B). We also observed that RA FLSs overexpressing THRIL exhibited significantly decreased migration and invasion rates than the control group (Figure $4 C, 4 D$ ). However, we found that the migration and invasion of $\mathrm{HC}$ FLSs with THRIL knockdown or overexpression were not different from that of the control siRNA or empty vector control group (Figure 4E-4H).

\section{Modulation of the expression of MMPs and proinflammatory cytokines by THRIL in RA FLSs}

RA FLSs have been found to secrete various MMPs in response to inflammatory stimulation, which directly degrade the extracellular matrix and facilitate invasion of RA FLSs into the articular cartilage and bones. Suppression of MMP expression might be an efficient strategy to block joint destruction. Interestingly, we found that silencing THRIL remarkably increased the mRNA expression of $M M P-1, M M P-3$, and $M M P-13$ in IL-1 $\beta$-treated RA FLSs (Figure 5A). However, THRIL overexpression resulted in a significant decrease in the IL- $1 \beta$-triggered expression of MMP-13 (Figure 5B). THRIL overexpression also slightly reduced the expression of $M M P-1$ and $M M P-3$ but had no statistical significance (Figure $5 B$ ). We also observed that silencing of THRIL increased the IL- $1 \beta$-triggered expression of MMP-1 mRNA in HC FLSs; however, we found that THRIL knockdown or overexpression did not affect the expression of $M M P-3$ and $M M P-13$ in IL-1 $\beta$ treated HC FLSs (Figure 5C,5D). In addition, we found that THRIL knockdown increased the expression of IL-6 and TNF- $\alpha, 2$ important pro-inflammatory cytokines in RA (Figure 5E).

\section{Downstream molecular targets of THRIL in RA FLSs}

To further explore how THRIL regulates RA FLSs function, 2 RT-PCR arrays designed to measure 84 genes related to the human cell cycle and 84 genes associated with human cell motility were used to identify target genes that are regulated by THRIL overexpression. As shown in Figure $6 A, 6 B$, a large panel of genes involved in cell proliferation and motility was found to be regulated by THRIL. The top 5 differentially expressed genes regulated by THRIL overexpression were further confirmed by qRTPCR in RA FLSs infected with THRIL overexpression lentivirus. We found that THRIL overexpression decreased the expression of MKI67, CDC20, CDK1, AURKB, and
GTSE1 (Figure 6C).

\section{Discussion}

In the current study, we reported that the lower-thannormal expression of lncRNA THRIL negatively regulated the proliferation rate, migration, and invasion of RA FLSs. In addition, THRIL modulated the expression of MMP-1, MMP-3, and MMP-13. Furthermore, we showed that THRIL regulated various genes associated with the cell cycle and cell motility. Our findings suggest that downregulated THRIL may contribute to excessive hyperplasia and invasive behaviors of rheumatoid pannus, ultimately leading to joint destruction.

It has been shown that some lncRNAs participate in RA pathogenesis $(10,21)$; however, their contribution to RA remains largely elusive. In the current investigation, we established decreased expression levels of IncRNA THRIL in RA FLSs compared to HC cells. THRIL knockdown or overexpression increased or decreased the migration, invasion, and proliferation rates of RA FLSs. Collectively, our data suggest that THRIL negatively regulates the tumor-like aggressiveness of RA FLSs. Our previous work demonstrated that the downregulated lncRNA LERFS negatively regulated migration and invasion by interacting with hnRNP Q in RA FLSs (12). Increasing evidence indicates an important role of FLSs in promoting rheumatoid synovial aggression $(3,22)$; therefore, our findings provide a potential novel target for controlling synovitis and joint damage in RA. Consistent with our results, THRIL was reported to be downregulated in gastric cancer tissues and non-small cell lung cancer tissues obtained from males compared with adjacent noncancerous tissues $(23,24)$. A recent report showed that THRIL modulated the migration and invasion of H9C2 cells (25). Another study indicated that THRIL was involved in the epithelial-to-mesenchymal transition phenotype of osteosarcoma cells (26).

MMPs, primarily secreted by FLSs in rheumatoid ST, are involved in the degradation of the extracellular matrix, leading to cartilage damage and bone erosion in RA joints. Pro-inflammatory cytokines, especially IL- $1 \beta$, are important inducers of MMP production. Nonetheless, the detailed mechanisms responsible for the expression of MMPs in RA FLSs are not well understood. Here, we found that THRIL knockdown or overexpression increased or decreased the expression of $M M P-13$ with IL-1 $\beta$ stimulation of RA FLSs. Our findings indicated that THRIL negatively regulates 


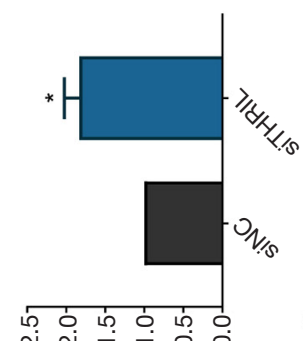

ஸึ ำ மீ

әреג ио!seли! әм!̣е|әу
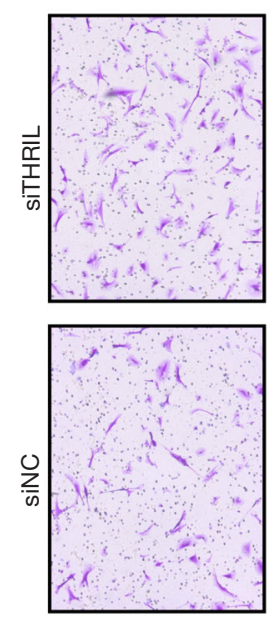

$\infty$

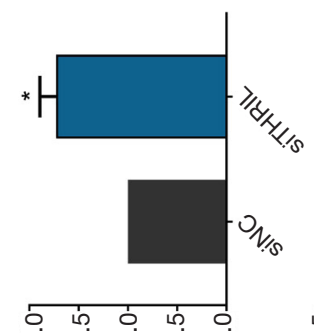

ஸे மீ.

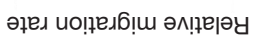
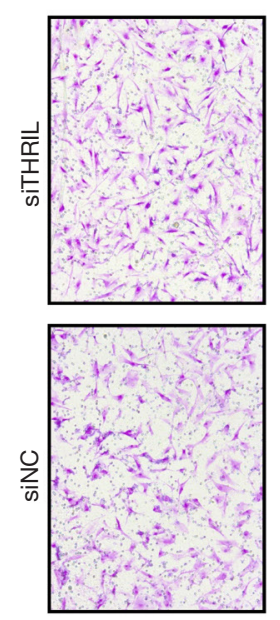

$\varangle$

uo!ıeı6!w

$\cup$

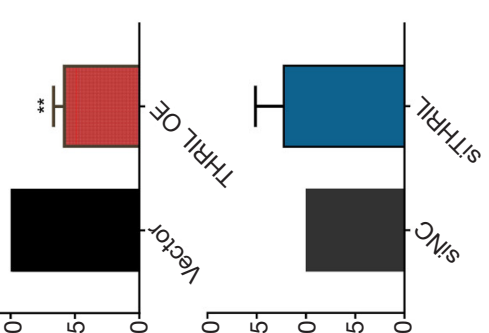

әрел ио!senu! әл!ฺ
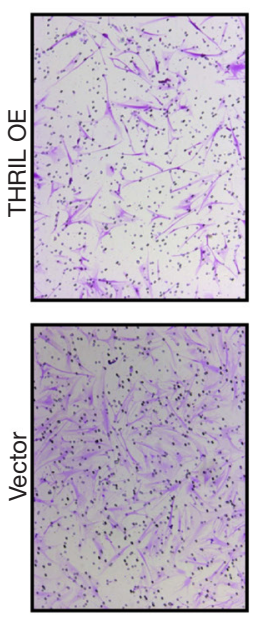

uọsenul

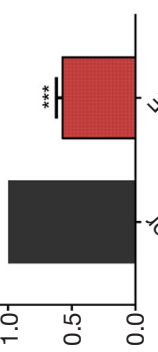

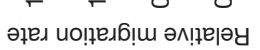
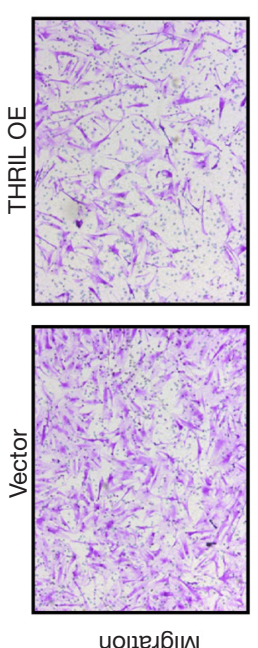
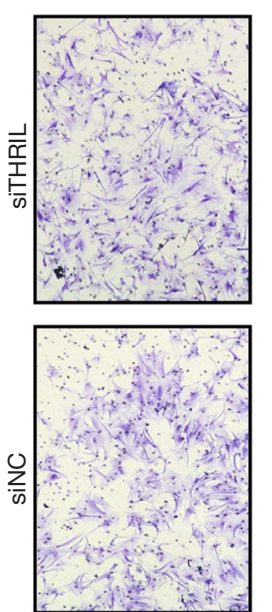

ㄴ
ค
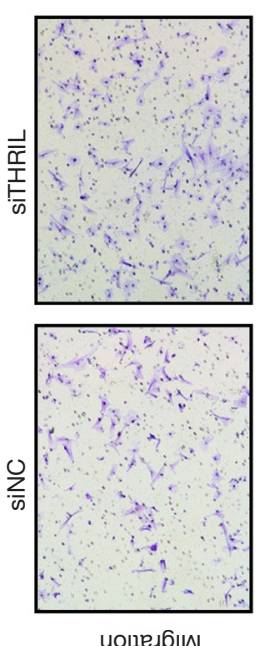

山

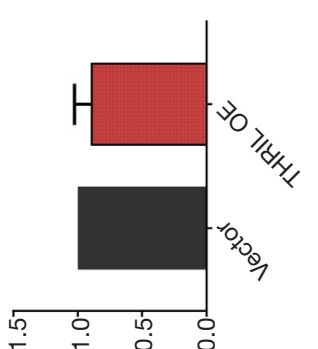

әрел ио!sеми! әм!ฺерәу
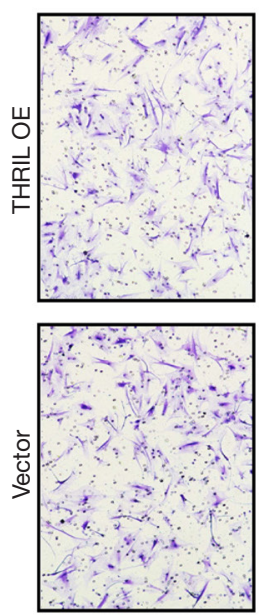

工
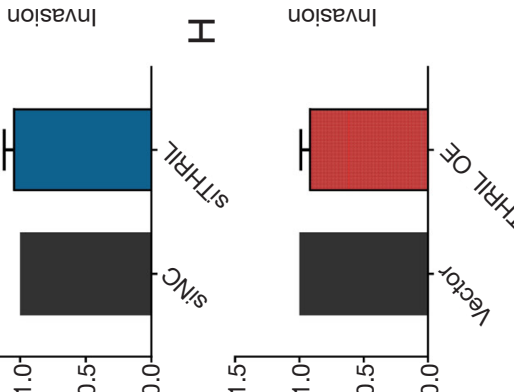
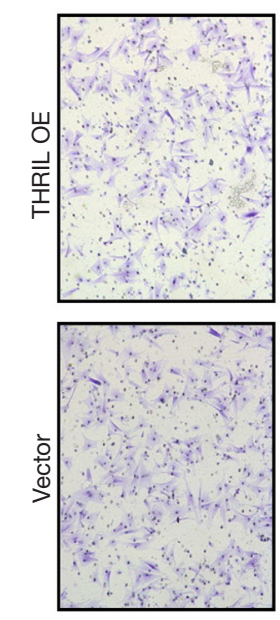

(
تี

.0.0.

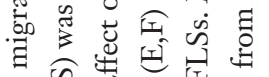

की

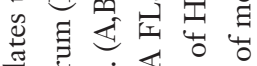

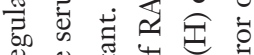

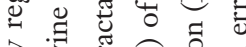

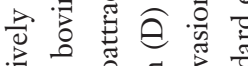

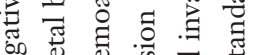

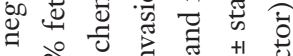

今웡

范范范

E

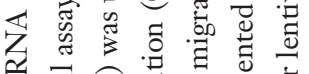

중

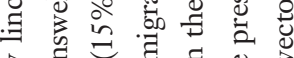

듑

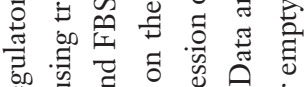

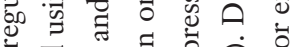

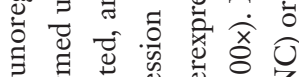

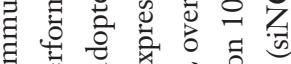

罗离要

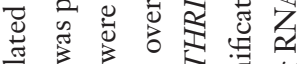

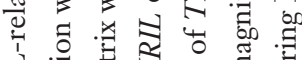

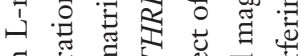

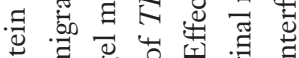

要

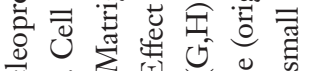

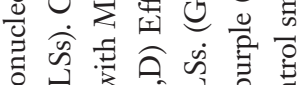

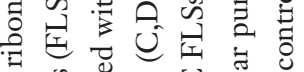

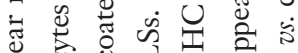

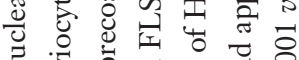

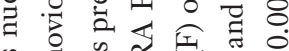

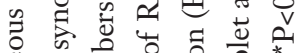

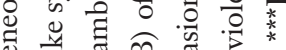

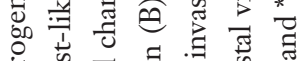

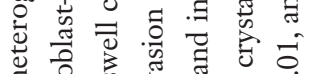

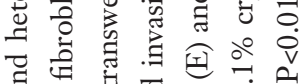

㟧

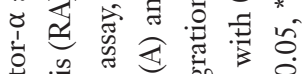

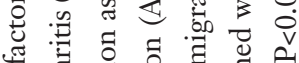

ษ

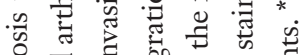

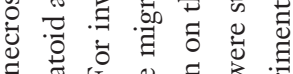

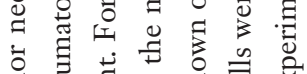

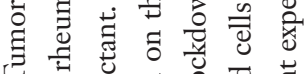

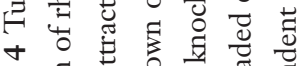

\%

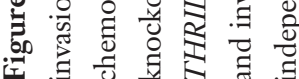


A

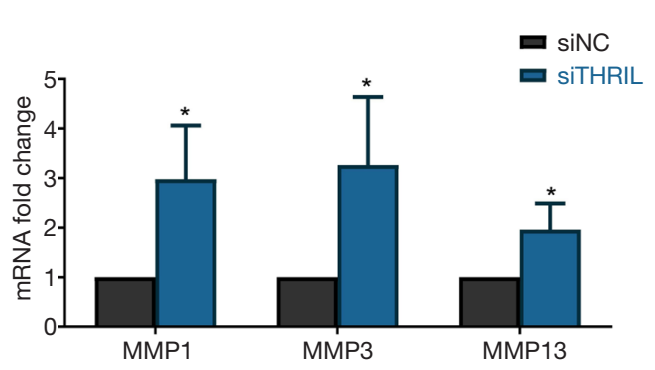

C

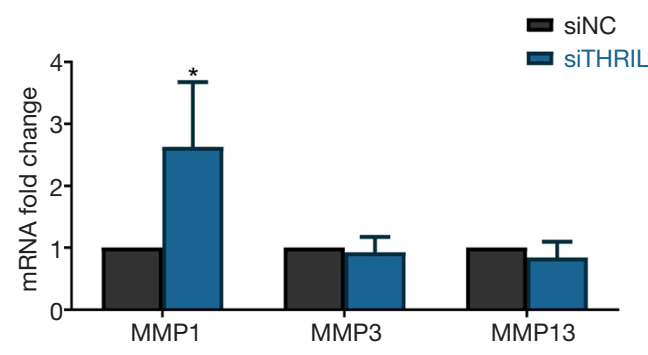

$\mathrm{E}$

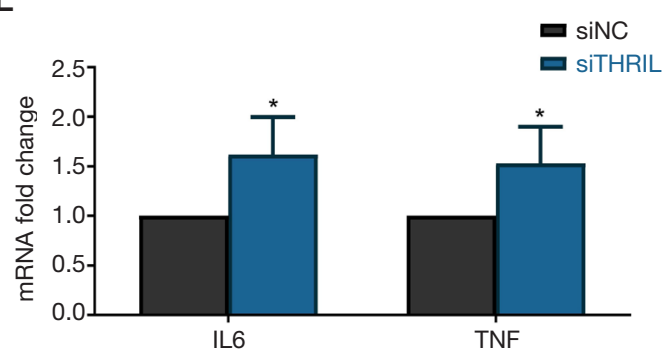

B

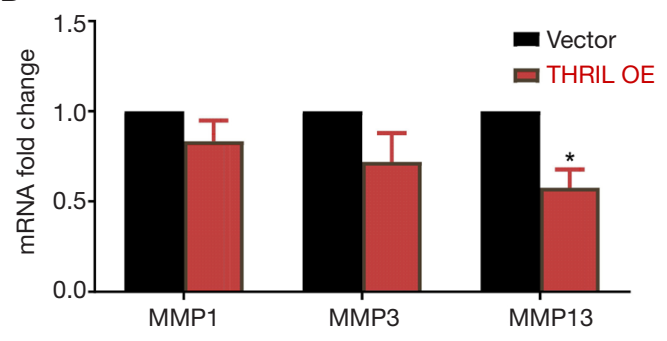

$\mathrm{D}$

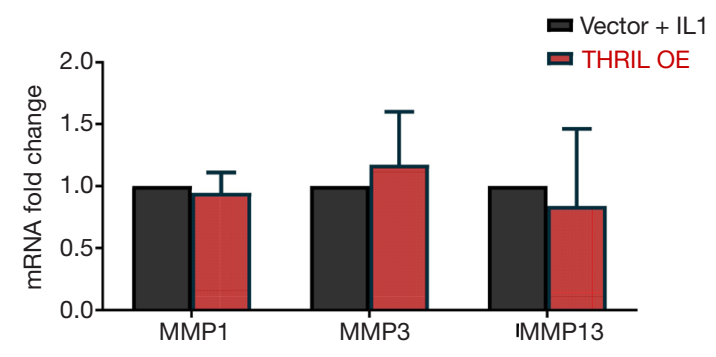

Figure 5 Modulation of interleukin (IL)-1 $\beta$-induced expression of matrix metalloproteinases (MMPs) and pro-inflammatory cytokines in rheumatoid arthritis (RA) fibroblast-like synoviocytes (FLS) by tumor necrosis factor- $\alpha$ (TNF- $\alpha$ ) and heterogeneous nuclear ribonucleoprotein L-related immunoregulatory lincRNA (THRIL). Cells were transfected with THRIL small interfering RNA (siTHRIL) or control siRNA (siNC) for $48 \mathrm{~h}$ or infected with THRIL overexpression lentivirus (THRIL OE) or empty vector lentivirus (Vector) and then stimulated with IL-1 $\beta(10 \mathrm{ng} / \mathrm{mL})$ for $24 \mathrm{~h}$. mRNA expression of MMPs and cytokines was quantified by quantitative reverse transcription polymerase chain reaction (qRT-PCR). (A,B) Effect of THRIL knockdown (A) or overexpression (B) on the expression of MMP-1, MMP-3, and MMP-13 in RA FLSs. (C,D) Effect of THRIL knockdown (C) or overexpression (D) on the expression of MMP-1, MMP-3, and MMP13 in HC FLSs. (E) Effect of THRIL knockdown on the expression of IL-6 and TNF- $\alpha$ in RA FLSs. Data are presented as mean \pm standard error of mean from at least 3 independent experiments. ${ }^{*} \mathrm{P}<0.05$ vs. siNC or Vector.

MMPs expression, which further supports the notion that THRIL is a powerful regulator in rheumatoid synovial aggression and joint damage.

In addition, we showed the suppressive effect on the proliferation of RA FLSs by THRIL. Similarly, a recent study indicated that silencing THRIL reversed TNF- $\alpha$ induced reduction of cell viability and enhancement of apoptosis (19). The unrestrained proliferation of resident FLSs is one of the leading contributors to the formation of rheumatoid pannus and joint destruction in RA; therefore, our findings suggest that the decreased level of THRIL in RA FLSs might be associated with abnormal synovial hyperplasia in RA. In line with our findings, it has been shown that THRIL is involved in the proliferation of endothelial progenitor cells and osteosarcoma cells $(26,27)$.

To further investigate the downstream molecular mechanisms and pathways through which THRIL modulates the pathogenic behaviors of RA FLSs, we employed real- 
A

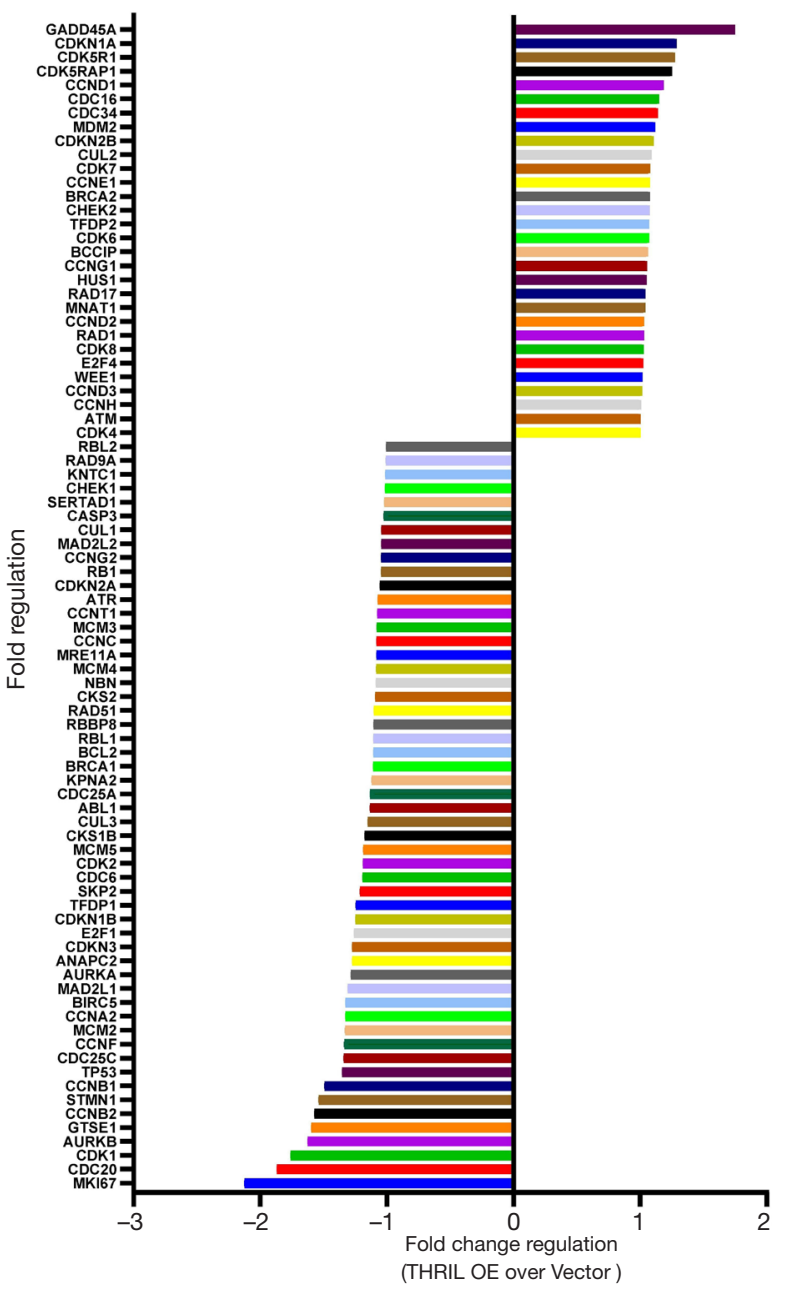

C

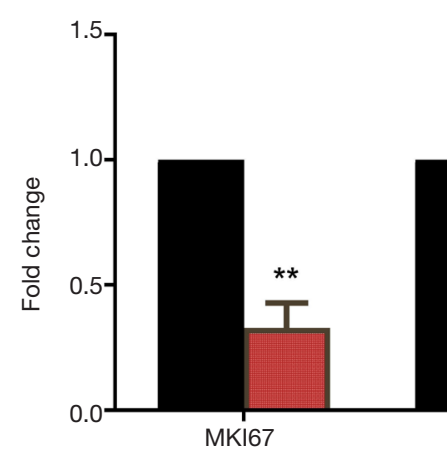

B

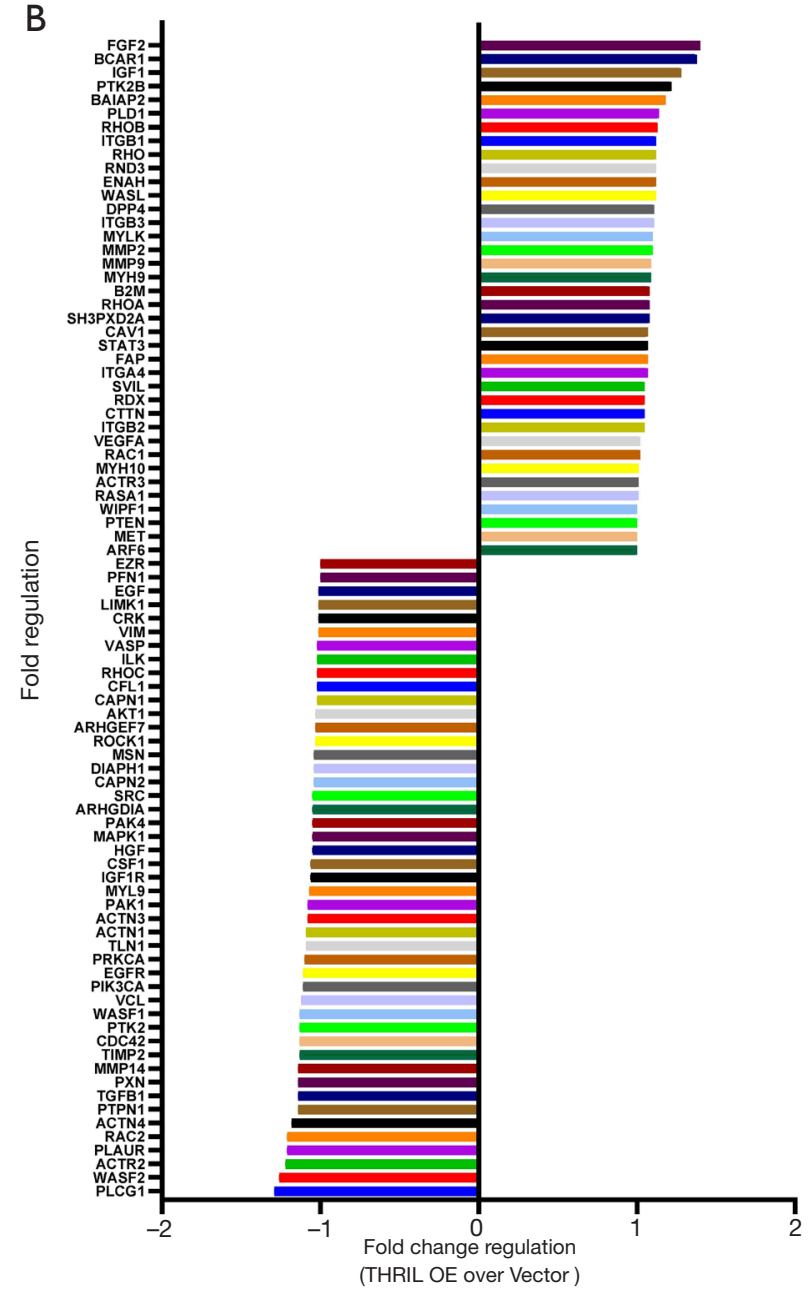

Vector

THRIL OE

Figure 6 Downstream target genes regulated by tumor necrosis factor- $\alpha$ and heterogeneous nuclear ribonucleoprotein L-related immunoregulatory lincRNA (THRIL). Rheumatoid arthritis (RA) fibroblast-like synoviocytes (FLSs) infected with control lentivirus (Vector) or THRIL overexpression lentivirus (THRIL OE) were subjected to human cell cycle real-time polymerase chain reaction (PCR) array (A) or human cell motility real-time PCR array (B). (C) Top 5 differentially expressed genes regulated by THRIL overexpression were further validated by quantitative reverse transcription polymerase chain reaction (qRT-PCR). The results are shown as mean \pm standard error of mean. ${ }^{*} \mathrm{P}<0.05,{ }^{* *} \mathrm{P}<0.01,{ }^{* * *} \mathrm{P}<0.001$, and ${ }^{* * * *} \mathrm{P}<0.0001$ vs. Vector. 
time PCR arrays to identify genes modulated by THRIL. A series of typical cell cycle-related genes were shown to be regulated by THRIL overexpression, which was further confirmed by qRT-PCR performed in RA FLSs infected with THRIL overexpression lentivirus. Among those differentially expressed genes, markers of proliferation Ki-67 (MKI67), cell division cycle 20 (CDC20), cyclindependent kinase $1(C D K 1)$, aurora kinase $\mathrm{B}(A U K R B)$, and G2 and S phase-expressed-1 (GTSE1) were confirmed to be downregulated by overexpression of THRIL. MKI67 is a nuclear protein required to disperse mitotic chromosomes during mitosis and proliferation (28). CDC20 and $A U K R B$ are important regulators participating in mitotic checkpoints and cell cycle regulation (29,30). CDK1, also known as $C D C 2$, a member of the cyclin-dependent kinase family, is essential for controlling the cell cycle and mitosis. Constitutive and deregulated CDK1 activation has been found to contribute to the aberrant proliferation of cancer cells by driving cell cycle progression $(31,32)$.

Interestingly, in addition to driving cell cycle progression, CDKs also carry out important roles in the modulation of the actin cytoskeleton and cell migration (33). Moreover, small-molecule inhibitors of CDKs are effective in treating experimental models of RA $(34,35)$, suggesting a potential treatment strategy for RA by targeting CDKrelated pathways. GTSE1 has been previously reported to contribute to cancer progression and metastasis by promoting cell proliferation and invasion $(36,37)$.

As previously reported, THRIL is a multifunctional lncRNA that has important roles in various cellular processes by transcriptional regulation of gene expression (17) and post-transcriptional regulation, such as targeting microRNAs $(25,38)$. Our data indicate that the modulation of THRIL in the invasion and proliferation of RA FLSs might be associated with its role in regulating the gene expression of CDK1 and GTSE1. Nevertheless, we do not rule out the possibility that some other mechanisms might be engaged in the regulation of RA FLSs by THRIL. Further studies are required to address the precise molecular mechanisms by which THRIL regulates the aggressive and proliferative behaviors of RA FLSs.

\section{Conclusions}

Our findings provide new evidence that the downregulated expression of THRIL negatively regulates the aggressive behavior of RA FLSs, suggesting that THRIL could be a novel key regulator controlling synovial hyperplasia and joint damage in RA.

\section{Acknowledgments}

We would like to thank all of the participants who were involved in the study.

Funding: This work was supported by the National Natural Science Foundation of China (grant No. 81801605, 81871275), Guangdong Natural Science Foundation (grant No. 2018A030313690), Guangdong Medical Scientific Research Foundation (grant No. A2018062), and Guangzhou Science and Technology Project (grant No. 201803010042).

\section{Footnote}

Reporting Checklist: The authors have completed the MDAR reporting checklist. Available at https://dx.doi. org/10.21037/atm-21-1362

Data Sharing Statement: Available at https://dx.doi. org/10.21037/atm-21-1362

Conflicts of Interest: All authors have completed the ICMJE uniform disclosure form (available at https://dx.doi. org/10.21037/atm-21-1362). The authors have no conflicts of interest to declare.

Ethical Statement: The authors are accountable for all aspects of the work in ensuring that questions related to the accuracy or integrity of any part of the work are appropriately investigated and resolved. All procedures performed in this study involving human participants were in accordance with the Declaration of Helsinki (as revised in 2013). All study procedures were approved by the Medical Ethics Committee of the First Affiliated Hospital of Sun Yat-sen University \{No. [2017]-049\}. Informed consent was provided by all patients.

Open Access Statement: This is an Open Access article distributed in accordance with the Creative Commons Attribution-NonCommercial-NoDerivs 4.0 International License (CC BY-NC-ND 4.0), which permits the noncommercial replication and distribution of the article with the strict proviso that no changes or edits are made and the original work is properly cited (including links to both the formal publication through the relevant DOI and the license). See: https://creativecommons.org/licenses/by-nc-nd/4.0/. 


\section{References}

1. Smolen JS, Aletaha D, McInnes IB. Rheumatoid arthritis. Lancet 2016;388:2023-38.

2. Alam J, Jantan I, Bukhari SNA. Rheumatoid arthritis: Recent advances on its etiology, role of cytokines and pharmacotherapy. Biomed Pharmacother 2017;92:615-33.

3. Bottini N, Firestein GS. Duality of fibroblast-like synoviocytes in RA: passive responders and imprinted aggressors. Nat Rev Rheumatol 2013;9:24-33.

4. Bartok B, Firestein GS. Fibroblast-like synoviocytes: key effector cells in rheumatoid arthritis. Immunol Rev 2010;233:233-55.

5. Nygaard G, Firestein GS. Restoring synovial homeostasis in rheumatoid arthritis by targeting fibroblast-like synoviocytes. Nat Rev Rheumatol 2020;16:316-33.

6. Guiducci G, Stojic L. Long Noncoding RNAs at the Crossroads of Cell Cycle and Genome Integrity. Trends Genet 2021;37:528-46.

7. Chew CL, Conos SA, Unal B, et al. Noncoding RNAs: Master Regulators of Inflammatory Signaling. Trends Mol Med 2018;24:66-84.

8. Schmitt AM, Chang HY. Long Noncoding RNAs in Cancer Pathways. Cancer Cell 2016;29:452-63.

9. Zou Y, Xu H. Involvement of long noncoding RNAs in the pathogenesis of autoimmune diseases. J Transl Autoimmun 2020;3:100044.

10. Lao MX, Xu HS. Involvement of long non-coding RNAs in the pathogenesis of rheumatoid arthritis. Chin Med J (Engl) 2020;133:941-50.

11. Taheri M, Eghtedarian R, Dinger ME, et al. Dysregulation of non-coding RNAs in Rheumatoid arthritis. Biomed Pharmacother 2020;130:110617.

12. Zou Y, Xu S, Xiao Y, et al. Long noncoding RNA LERFS negatively regulates rheumatoid synovial aggression and proliferation. J Clin Invest 2018;128:4510-24.

13. Yu H, Ding C, Dai S, et al. Long noncoding RNA FER1L4 regulates rheumatoid arthritis via targeting NLRC5. Clin Exp Rheumatol 2020;38:713-23.

14. Ye Y, Gao X, Yang N. LncRNA ZFAS1 promotes cell migration and invasion of fibroblast-like synoviocytes by suppression of miR-27a in rheumatoid arthritis. Hum Cell 2018;31:14-21.

15. Mo BY, Guo XH, Yang MR, et al. Long Non-Coding RNA GAPLINC Promotes Tumor-Like Biologic Behaviors of Fibroblast-Like Synoviocytes as MicroRNA Sponging in Rheumatoid Arthritis Patients. Front Immunol 2018;9:702.
16. Bi X, Guo XH, Mo BY, et al. LncRNA PICSAR promotes cell proliferation, migration and invasion of fibroblast-like synoviocytes by sponging miRNA-4701-5p in rheumatoid arthritis. EBioMedicine 2019;50:408-20.

17. Li Z, Chao TC, Chang KY, et al. The long noncoding RNA THRIL regulates TNF expression through its interaction with hnRNPL. Proc Natl Acad Sci U S A 2014;111:1002-7.

18. Moharamoghli M, Hassan-Zadeh V, Dolatshahi E, et al. The expression of GAS5, THRIL, and RMRP lncRNAs is increased in T cells of patients with rheumatoid arthritis. Clin Rheumatol 2019;38:3073-80.

19. Liang Y, Li H, Gong X, et al. Long Non-coding RNA THRIL Mediates Cell Growth and Inflammatory Response of Fibroblast-Like Synoviocytes by Activating PI3K/AKT Signals in Rheumatoid Arthritis. Inflammation 2020;43:1044-53.

20. Aletaha D, Neogi T, Silman AJ, et al. 2010 Rheumatoid arthritis classification criteria: an American College of Rheumatology/European League Against Rheumatism collaborative initiative. Arthritis Rheum 2010;62:2569-81.

21. Wang J, Yan S, Yang J, et al. Non-coding RNAs in Rheumatoid Arthritis: From Bench to Bedside. Front Immunol 2019;10:3129.

22. Mousavi MJ, Karami J, Aslani S, et al. Transformation of fibroblast-like synoviocytes in rheumatoid arthritis; from a friend to foe. Auto Immun Highlights 2021;12:3.

23. Esfandi F, Salehnezhad T, Taheri M, et al. Expression assessment of a panel of long non-coding RNAs in gastric malignancy. Exp Mol Pathol 2020;113:104383.

24. Esfandi F, Taheri M, Omrani MD, et al. Expression of long non-coding RNAs (lncRNAs) has been dysregulated in non-small cell lung cancer tissues. BMC Cancer 2019;19:222.

25. Xia J, Jiang N, Li Y, et al. The long noncoding RNA THRIL knockdown protects hypoxia-induced injuries of H9C2 cells through regulating miR-99a. Cardiol J 2019;26:564-74.

26. Xu B, Jin X, Yang T, et al. Upregulated lncRNA THRIL/ TNF- $\alpha$ Signals Promote Cell Growth and Predict Poor Clinical Outcomes of Osteosarcoma. Onco Targets Ther 2020;13:119-29.

27. Xiao J, Lu Y, Yang X. THRIL mediates endothelial progenitor cells autophagy via AKT pathway and FUS. Mol Med 2020;26:86.

28. Cuylen S, Blaukopf C, Politi AZ, et al. Ki-67 acts as a biological surfactant to disperse mitotic chromosomes. Nature 2016;535:308-12. 
29. Izawa D, Pines J. The mitotic checkpoint complex binds a second CDC20 to inhibit active APC/C. Nature 2015;517:631-4.

30. Kelly JR, Martini S, Brownlow N, et al. The Aurora $\mathrm{B}$ specificity switch is required to protect from nondisjunction at the metaphase/anaphase transition. Nat Commun 2020;11:1396.

31. Malumbres M, Barbacid M. Cell cycle, CDKs and cancer: a changing paradigm. Nat Rev Cancer 2009;9:153-66.

32. Malumbres M. Cyclin-dependent kinases. Genome Biol 2014;15:122.

33. Hydbring P, Malumbres M, Sicinski P. Non-canonical functions of cell cycle cyclins and cyclin-dependent kinases. Nat Rev Mol Cell Biol 2016;17:280-92.

34. Hosoya T, Iwai H, Yamaguchi Y, et al. Cell cycle regulation therapy combined with cytokine blockade

Cite this article as: Zou Y, Shen C, Shen T, Wang J, Zhang X, Zhang Q, Sun R, Dai L, Xu H. LncRNA THRIL is involved in the proliferation, migration, and invasion of rheumatoid fibroblast-like synoviocytes. Ann Transl Med 2021;9(17):1368. doi: 10.21037/atm-21-1362 enhances antiarthritic effects without increasing immune suppression. Ann Rheum Dis 2016;75:253-9.

35. Sekine C, Sugihara T, Miyake S, et al. Successful treatment of animal models of rheumatoid arthritis with smallmolecule cyclin-dependent kinase inhibitors. J Immunol 2008;180:1954-61.

36. Guo L, Zhang S, Zhang B, et al. Silencing GTSE1 expression inhibits proliferation and invasion of hepatocellular carcinoma cells. Cell Biol Toxicol 2016;32:263-74.

37. Lin F, Xie YJ, Zhang XK, et al. GTSE1 is involved in breast cancer progression in p53 mutation-dependent manner. J Exp Clin Cancer Res 2019;38:152.

38. Chen H, Hu X, Li R, et al. LncRNA THRIL aggravates sepsis-induced acute lung injury by regulating miR-424/ ROCK2 axis. Mol Immunol 2020;126:111-9. 